\title{
Dengue Fever in Adults, a Retrospective Study
}

\author{
Mohammed Salah Debes ${ }^{1,}$, , Mohamed Al Tayeb², Momen Faysal Nassani², \\ Ahmed Abdullah Basaeed ${ }^{2}$, Anas Dwaima ${ }^{2}$, Moahmmed Khaldoun Alsaadi ${ }^{2}$, Hassan Odah ${ }^{2}$ \\ ${ }^{1}$ School of Clinical Sciences, University of Bristol, Bristol, UK \\ ${ }^{2}$ Department of Internal Medicine, International Medical Centre, Jeddah, Saudi Arabia
}

Email address:

mohammad.debes@hotmail.com (M. S. Debes)

${ }^{*}$ Corresponding author

\section{To cite this article:}

Mohammed Salah Debes, Mohamed Al Tayeb, Momen Faysal Nassani, Ahmed Abdullah Basaeed, Anas Dwaima, Moahmmed Khaldoun Alsaadi, Hassan Odah. Dengue Fever in Adults, a Retrospective Study. American Journal of Internal Medicine. Vol. 4, No. 6, 2016 , pp. 93-100. doi: 10.11648/j.ajim.20160406.11

Received: April 8, 2016; Accepted: July 19, 2016; Published: November 10, 2016

\begin{abstract}
Background: Dengue fever is a very common viral infection with potential serious complications and is one of the WHO challenges to limit its spread especially in view of lacking specific treatment or approved vaccine. We noticed over long time frequent cases of dengue fever with some characteristic laboratory findings particularly leukopenia, thrombocytopenia and elevated liver enzymes. These findings sometimes give concerns about safety of the patients so admission and sometimes isolation that could be unnecessary for some patients. Objectives: To present our data about variable clinical presentations and laboratory changes associated with dengue fever infection in adults and complications encountered in our institute in Saudi Arabia. So that we can give recommendations about the diagnosis, management and protection. Methods: We included all dengue fever positive cases starting September 2007 till end of December 2014. The data gathered through tracking the records of patients requested for Dengue fever serology in the main laboratory of the International Medical Centre (IMC). Data for analysis: Age, gender, characteristic laboratory changes, clinical manifestations and complications. Results: Dengue fever is a self-limiting viral infection in the majority of cases. Incidence of dengue infection in males: females is 2:1. Dengue fever manifestations in order of frequency: Fever, body aches, gastro intestinal symptoms, headache, and skin rash and chest symptoms. Incidence of complications in our study was $5.5 \%$.
\end{abstract}

Keywords: Dengue, Fever, Virus, Infection, Manifestations of Dengue Fever

\section{Introduction}

Dengue fever is a viral infection endemic in tropical and subtropical areas [1], [15]. Its incidence and distribution on the map dramatically changed over the last decades [24] which makes it a global health concern. It is caused by dengue virus which is a single stranded RNA virus belongs to the family flaviviridae with four recognized serotypes (DENV1 to DENV4) [1], [2], [13]. The four serotypes can cause classic dengue fever DF and dengue hemorrhagic fever DHF [29]. Infection with one serotype confers lifelong immunity against this particular serotype but the patient is still liable for infection with the other serotypes and even secondary infection has more risk of complications [3].

Transmission via bite of the mosquito Aedes Aegypti which is the main vector [36] and affects millions of people annually
[8] so it is considered the most prevalent mosquito born infection worldwide [20].

The Aedes mosquito (male and female) feeds on nectar of plants but females in need for feeding on blood to lay eggs so the female mosquito is the main vector and it primarily bites humans [9]. Dengue spread was also reported via Aedes albopictus [7], [10], [19]. The mosquito is mainly active during day time [11] but the bite may pass unnoticed. After feeding on blood of a carrier it takes about 3-14 days incubation period for the virus [10] then mosquitoes lay eggs above the water line in stagnant water like water containers [9]. The eggs are resistant to dryness and may survive for months and this capability to survive for long time would increase possibility of spread to other locations [9]. Aedes Aegypti in particular has the tendency to bite frequently in a single gonotropic cycle [26] also infected mosquitoes have longer 
feeding duration [27] and this increases the likelihood to gain and subsequently transmit more than one serotype [23] which increases dramatically virus spread [26].

\section{Methodology and Materials}

As health care providers in Saudi Arabia we encounter many cases of Dengue fever so we ran this study retrospectively looking for the various presentations, laboratory changes and possible complications of dengue fever infection.

\subsection{Diagnosis of Dengue}

We based the diagnosis on positive dengue serology IgM or anti nonstructural protein 1 (NS1) in our institute as well as positive PCR in the central laboratory of the $\mathrm{MOH}$ which goes with the literature way for Dengue diagnosis [30], [35].

\subsection{Inclusion Criteria}

-Confirmed dengue cases.

-Cases that were followed in our institute so that at least two $\mathrm{CBC}$ results available.

-No other confounding factors such as co-infection that may alter the clinical and/or the laboratory results.

\subsection{Exclusion Criteria}

-Negative cases for dengue serology.

-Patients with less than two CBC readings.

-Cases with any co-morbidity that may alter the laboratory and/or clinical findings such as other viral infection.

\subsection{Data Gathering}

We did trace all dengue fever tests done in our institute starting 2007 till end of December 2014. We reviewed 3277 cases requested for Dengue fever. Positive cases were taken then we excluded 180 positive cases including cases due to co-infection with other pathogens like malaria, CMV, EBV, HAV\&HCV also those with co-incident bacterial infections were excluded as the hematologic changes would be affected by these co-infections. Also excluded cases with bone marrow suppression, known cases of liver cirrhosis, cases received blood transfusion and a case with acute pancreatitis as all these scenarios would also alter hematological findings, but the vast majority of excluded cases were due to lack of follow up so they did not meet our inclusion criteria.

As the dengue fever mainly affects the bone marrow (CBC) and liver so our main data tracing focused on these two items to find out the different patterns of changes during the course of infection as well as data about various clinical presentations and complications encountered.

Hematological parameters:

Total white blood cell count (WBCs): WBCs count change showed variable patterns ranging from normal WBCs to leukopenia and also leukocytosis so we took the lowest figure in cases with leukopenia and the highest in cases with leukocytosis.

Neutrophil count (ANC) we took the lowest figure in leukopenia and the highest in leukocytosis.
Lymphocyte count we took the lymphocyte count of the same CBC of ANC (same day of ANC).

Monocyte percent we took the highest monocyte percent out of all CBCs done during the dengue follow up course.

Platelet count the platelet count change ranged between normal platelet counts to thrombocytopenia with no cases of thrombocytosis encountered so we took the lowest platelet count out of all CBCs done during the dengue follow up course.

Liver enzymes we took the ALT as the prototype for parenchymal liver injury and we reported the highest figure out of all LFTs done during dengue course. We considered significant increment of ALT when the figure is at least two folds the high normal. So according to our laboratory reference it is considered significant when ALT is $90 \mathrm{mg} / \mathrm{dl}$ or more.

Then the recovery time:

The recovery time means the time interval from start of laboratory changes until the curve of changes going back to the improvement not the time of full improvement back to normal.

We took the recovery time as follows:

WBCs recovery means start of the slope to shift back to normal so cases with leukopenia recovery considered when WBCs start to increase. Cases with leukocytosis recovery considered when WBCs start to decrease.

These parameters taken as a sign of hematological recovery.

Platelets recovery also refers to the figure at which the curve starts to correct toward normal. So in cases with thrombocytopenia we consider the figure when platelets count starts to increase. Also considered as a sign of hematologic recovery. But we do not have cases with thrombocytosis.

ALT recovery when ALT figure starts to decrease after significant increment.

\section{Statistical Analysis and Results}

We came up with 496 positive dengue cases, 329 males which is $66.33 \%$ and 167 females which is $33.6 \%$ of the total. So the incidence of dengue infection males to females is $2: 1$. Then we divided the selected cases according to gender and we did comparison between the two groups using student t-test.

\subsection{Haematological Derangement}

Table 1. Table of hematological changes.

\begin{tabular}{|c|c|c|c|c|c|}
\hline Item & $\begin{array}{l}\text { Mean for } \\
\text { males }\end{array}$ & SD & $\begin{array}{l}\text { Mean for } \\
\text { Females }\end{array}$ & SD & Pvalue \\
\hline Total WBCs & 3.23 & 2.5 & 3.26 & 3.43 & 0.936 \\
\hline \multicolumn{6}{|c|}{ Then divided WBCs change manner into three categories } \\
\hline Normal WBCs & 5.58 & 1.38 & 5.88 & 1.91 & 0.445 \\
\hline Leukocytosis & 14.3 & 2.3 & 17.98 & 8.92 & 0.23 \\
\hline Leukopenia & 2.31 & 0.72 & 2.22 & 0.739 & 0.20 \\
\hline \multicolumn{6}{|c|}{ Differential WBCs } \\
\hline ANC & 1.76 & 4.29 & 1.67 & 2.72 & 0.80 \\
\hline Lymphocytes & 1.28 & 1.068 & 1.314 & 1.154 & 0.80 \\
\hline Monocytes & 15.05 & 5.99 & 11.93 & 3.82 & 0.0001 \\
\hline \multicolumn{6}{|c|}{ Platelets total and differential } \\
\hline Total & 98.8 & 57.82 & 116.26 & 61.56 & 0.0023 \\
\hline Low platelets & 83.62 & 36.96 & 89.8 & 38.44 & 0.127 \\
\hline Normal & 192.82 & 34.06 & 201.57 & 42.16 & 0.321 \\
\hline
\end{tabular}




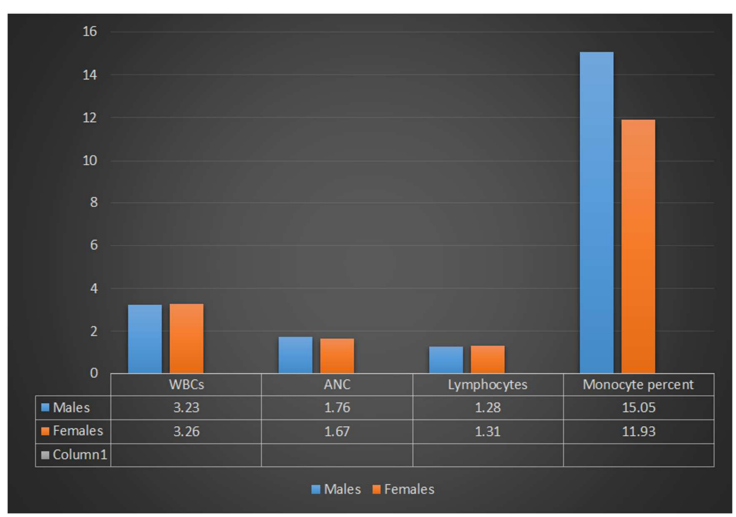

Figure 1. WBCS changes chart showing mean of changes both males and females.

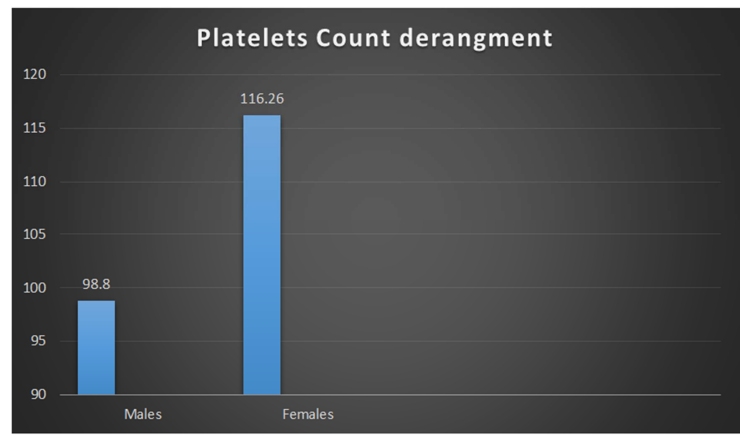

Figure 2. Mean of Platelet count derangement (males \& females)

\subsection{Liver Enzyme Derangement}

Table 2. ALT changes (males \& females).

\begin{tabular}{llllll}
\hline Item & $\begin{array}{l}\text { Mean for } \\
\text { males }\end{array}$ & SD & $\begin{array}{l}\text { Mean for } \\
\text { Females }\end{array}$ & SD & P value \\
\hline ALT & 132.93 & 366.62 & 165.43 & 785.85 & 0.55 \\
$\begin{array}{l}\text { Significant ALT } \\
\text { elevation }>90 ~ \mathrm{mg} / \mathrm{dl}\end{array}$ & 289.9 & 573.3 & 387.18 & 1287.2 & 0.50 \\
\hline
\end{tabular}

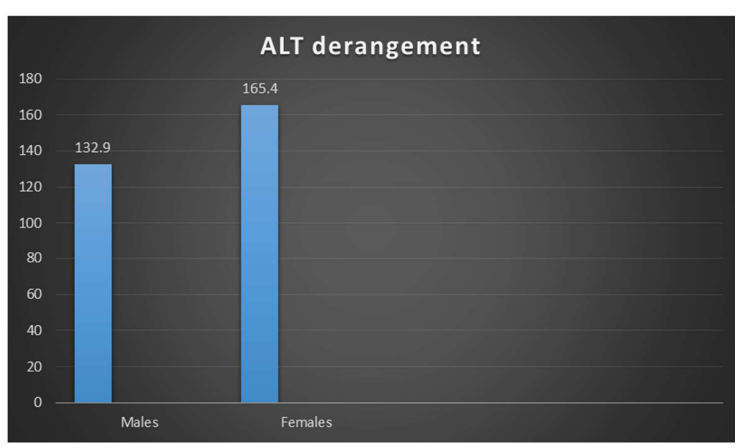

Figure 3. Mean of ALT derangement (males \& females).

\subsection{Incidence}

Table 3. Incidence of Hematological and liver enzyme alterations.

\begin{tabular}{lll}
\hline Item & males & Females \\
\hline Normal WBCs & $17.3 \%$ & $13.2 \%$ \\
Leucocytosis & $3.03 \%$ & $3.01 \%$ \\
Leukopenia & $79.9 \%$ & $82.5 \%$ \\
Low platelets & $87.23 \%$ & $73.49 \%$ \\
Normal platelets & $11.85 \%$ & $26.51 \%$ \\
Significant ALT rise & $36.66 \%$ & $36.0 \%$ \\
\hline
\end{tabular}

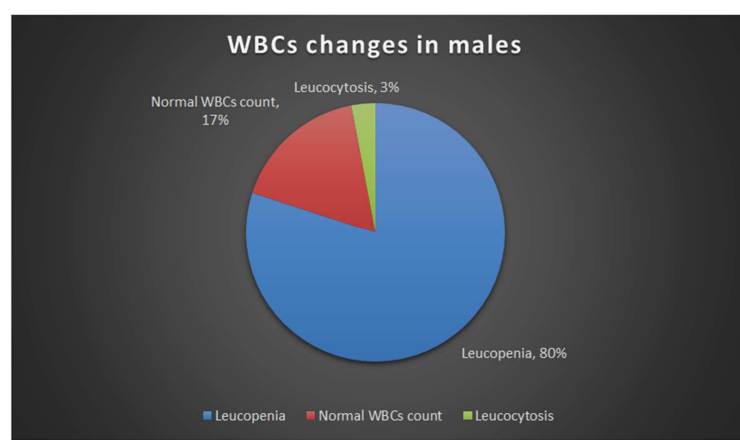

Figure 4. WBCs changes in males.

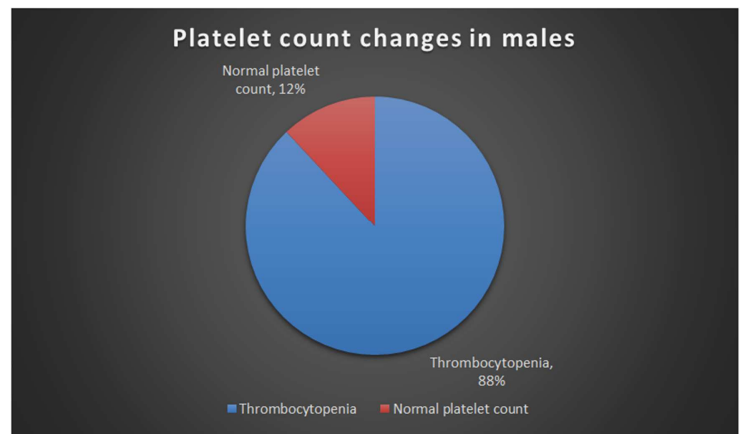

Figure 5. Platelet count changes in males.

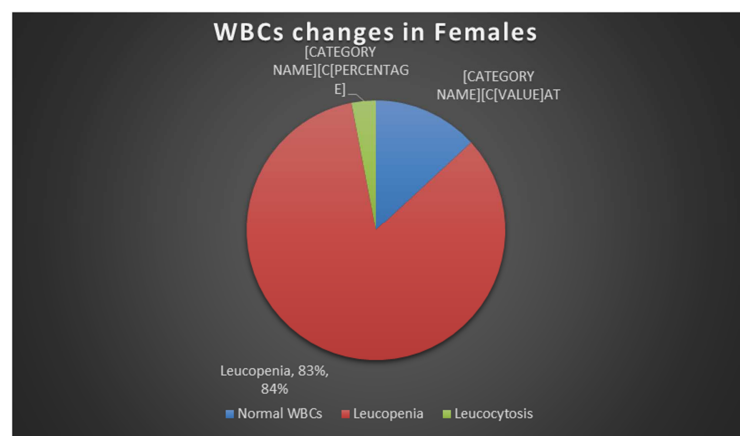

Figure 6. WBCs changes in females.

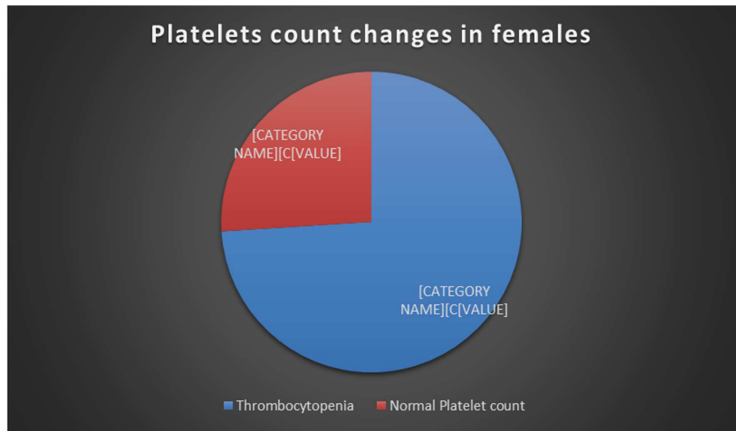

Figure 7. Platelet count changes in females.

\subsection{Cases with Marked Derangement}

Table 4. Patterns of marked derangement of Platelets, ANC and ALT.

\begin{tabular}{llllll}
\hline Item & $\begin{array}{l}\text { Mean for } \\
\text { males }\end{array}$ & SD & $\begin{array}{l}\text { Mean for } \\
\text { Females }\end{array}$ & SD & $\begin{array}{l}\text { P } \\
\text { value }\end{array}$ \\
\hline Platelets $<50$ & 28.44 & 15.2 & 23.86 & 13.97 & 0.234 \\
ALT $>$ tenfold & 1437.13 & 1819.27 & 3365.14 & 4251.12 & 0.2627 \\
ANC $<0.5$ & 0.312 & 0.133 & 0.299 & 0.113 & 0.648 \\
\hline
\end{tabular}




\subsection{Incidence of Marked Derangement}

Table 5. Incidence of cases with marked derangement.

\begin{tabular}{lll}
\hline Item & males & Females \\
\hline Platelets $<50$ & $16.41 \%$ & $12.65 \%$ \\
ALT $>$ tenfold & $2.66 \%$ & $4.66 \%$ \\
ANC $<0.5$ & $19.14 \%$ & $17.46 \%$ \\
\hline
\end{tabular}

\subsection{Recovery Time}

Table 6. Recovery time of hematologic and ALT alterations.

\begin{tabular}{llllll}
\hline Item & $\begin{array}{l}\text { Mean for } \\
\text { males }\end{array}$ & SD & $\begin{array}{l}\text { Mean for } \\
\text { Females }\end{array}$ & SD & P value \\
\hline Platelets & 4.42 & 2.65 & 3.85 & 2.03 & 0.03 \\
WBCs & 3.28 & 1.9 & 3.59 & 2.65 & 0.292 \\
ALT & 4.44 & 4.26 & 3.77 & 2.43 & 0.4271 \\
\hline
\end{tabular}

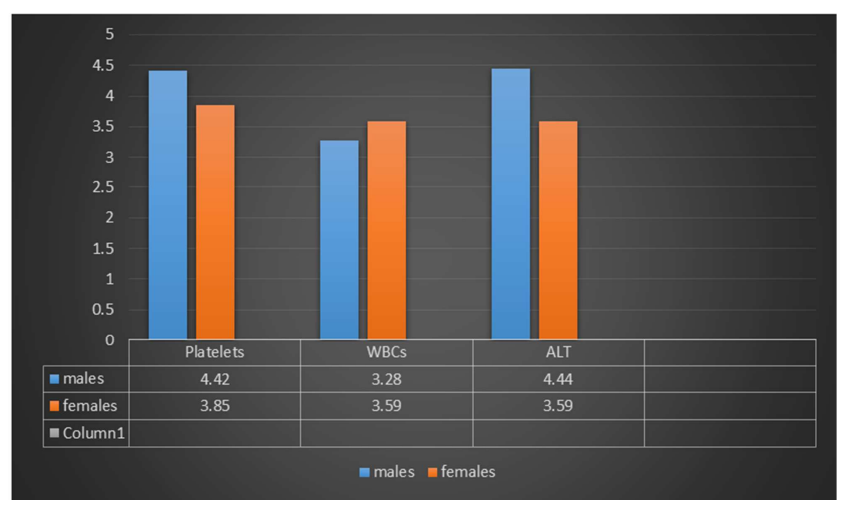

Figure 8. Recovery time chart.

So in our data:

Different patterns of WBCs changes with leucopenia the most common but still normal leucocyte count and leukocytosis are part of the spectrum. Also Platelet count mostly drops giving striking picture in the context of fever that is usually the clue to prompt clinicians to check for dengue but still normal platelet count is encountered in $11.6 \%$ in males and $26.5 \%$ in females. The ALT is elevated in about one third of cases but incidence of marked elevation (exceeding ten folds) is about $2.6 \%$ in males and $4.6 \%$ in females. No significant difference between males and females in most parameters with exception of: Monocyte percent as males showed higher monocyte percent and the difference was statistically significant. Also platelets count showed statistically significant difference with males showing more thrombocytopenia.

The recovery time for these laboratory changes averaged 4 days and it was comparable in both genders with exception of thrombocytopenia recovery time that was longer in males with statistically significant difference.

There was observed time lag between ALT changes and the hematologic changes as the ALT starts to rise and recovers later than the hematologic derangement and this applies for both genders.

\subsection{Clinical Manifestations}

Here we present the different clinical manifestations encountered but the number of cases is less than those included in the laboratory tracing and this will be explained later.

Males: 184 cases traced

Age: mean age 38.66 with SD 14.84

Fever 184 so $100 \%$ with mean temp 38.155 and SD 0.935

Skin manifestations (rash, itchiness): 39 cases so $21.1 \%$

Gastro intestinal (GI) manifestations (nausea, vomiting, diarrhea): 53 cases so $28.8 \%$

Headache/retro-orbital pain: 44 cases so $23.9 \%$

Body aches: 108 cases so $58.69 \%$

Chest symptoms (cough dry/productive): 19 cases so $10.3 \%$ Females: 81 cases

Age: mean age 35.16 with SD 14.03

Fever 81 so $100 \%$ with mean temp 38.332 and SD 0.954 .

Skin manifestations (rash, itchiness): 24 cases so $29.6 \%$

GI manifestations (nausea, vomiting, diarrhea): 39 cases so $48.1 \%$

Headache/retro-orbital pain: 27 cases so $33.3 \%$

Body aches: 51 cases so $62.9 \%$

Chest symptoms (cough dry/productive): 9 cases so $11.1 \%$

Table 7. Clinical Manifestations.

\begin{tabular}{lllll}
\hline Clinically & Males & & Females & \\
\hline Age & 36.98 & SD 14.4 & 35.16 & SD 13.8 \\
Temperature & 38.15 & SD 0.935 & 38.98 & SD 0.48 \\
Fever & $100 \%$ & & $100 \%$ & \\
Skin (rash, itchiness) & $21.1 \%$ & & $29.6 \%$ & \\
GI symptoms: & $28.8 \%$ & & $48.1 \%$ & \\
(nausea, vomiting, diarrhea) & $23.9 \%$ & & $33.3 \%$ & \\
Headache & $58.69 \%$ & & $62.9 \%$ & \\
Body aches & $10.3 \%$ & & $11.1 \%$ & \\
Chest symptoms (cough) & & & & \\
\hline
\end{tabular}

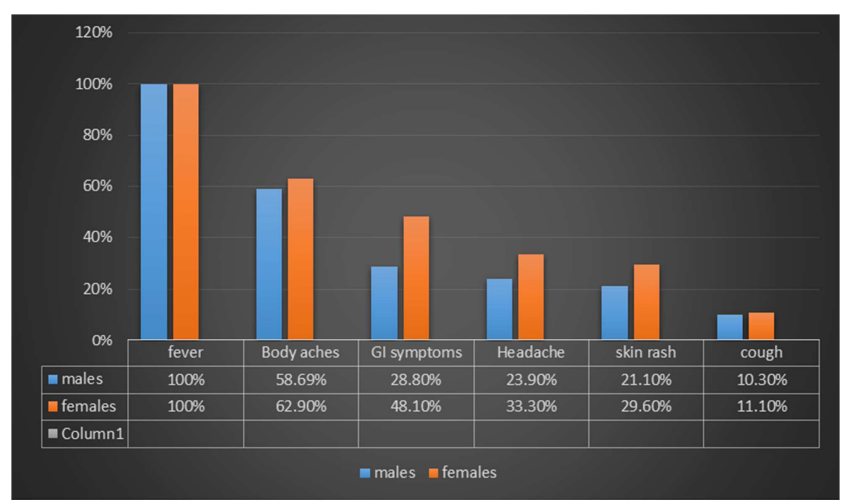

Figure 9. Chart of clinical Manifestations.

\subsection{Complications Encountered}

One case had menorrhagia.

One case had hematemesis and put on PPI

Two cases dehydrated.

One case fulminant liver failure and transferred to liver center.

One case had psychiatric manifestations: anxiety, irritability, crying.

One case acute renal failure (ARF).

One case admitted to ICU with seizures and diagnosed as 
dengue encephalitis.

Two cases melena, upper GI scope revealed bleeding PUD.

Two cases had gum bleeding.

Also bruises in one case and given platelets.

One case hematuria.

Two cases had hypotension and pre-syncope.

So Overall complications rate in our study is $5.55 \%$ as follows:

-Bleeding (menorrhagia, hematemesis, melena, hematuria, bruises) in $2.64 \%$. So $47 \%$ of overall complications.

-Dehydration, ARF, hypotension and pre-syncope in $1.8 \%$. So $32 \%$ of overall complications.

-Neuropsychiatric manifestations such as crying, irritability and anxiety $0.74 \%$. So $13 \%$ of overall complications.

-Fulminant liver failure in $0.37 \%$. So $6.6 \%$ of overall complications.

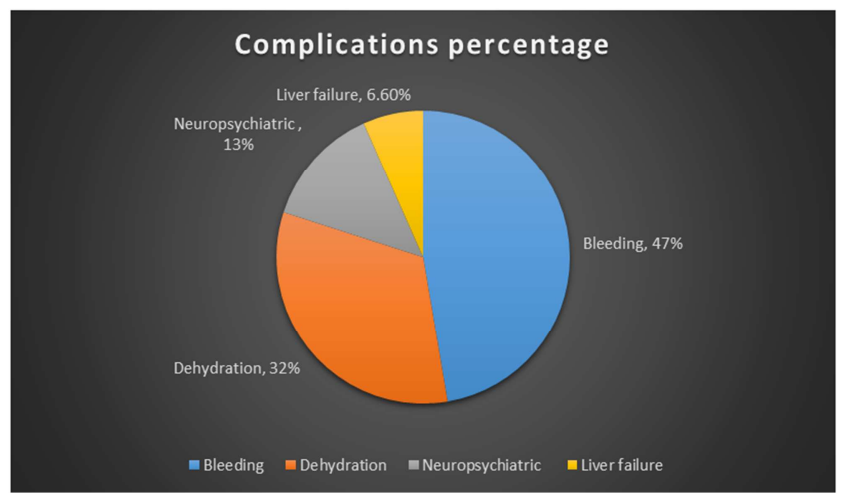

Figure 10. Percentage of complications.

\section{Discussion}

In our report the incidence males: females is $2: 1$ and same incidence was reported in Dar es Salaam 2014 outbreak [30] and this could be due to the dressing of Muslim females in the KSA which would give some protection against mosquito bites.

Dengue fever or break bone fever [12] has three phases febrile, critical then recovery [36]. Incubation period ranges from 2-7 days [13] followed by acute onset of fever that may range from being asymptomatic to symptomatic fever [7], [34], [35] with diffuse body aches, arthralgia, myalgia, headache and rash. In our report manifestations in order of frequency: Fever, body aches, gastro intestinal symptoms such as nausea, vomiting or diarrhea, headache mainly retro-orbital pain but could also be diffuse, skin rash that is centrifugal maculopapular erythematous and sometimes itchy and the least frequently encountered chest symptoms which are sometimes so significant leading to mis-diagnosis as chest infection.

In our study the recovery time was average of 3-5 days for hematological and liver enzyme changes going with the literature data about viremia average of 5 days [23], [28] which is also positively correlated with the clinical manifestations as at the time viremia subsides, fever settles and symptoms would start to resolve [29], [31].

Incidence of complications in our report is $5.5 \%$ with the hemorrhagic complications accounting for $47 \%$ of all complications. In the literature despite no reporting of hemorrhage percent among overall complications but still severe form of this entity is well reported as potentially fatal hemorrhagic dengue fever known as infectious thrombocytopenic purpura when platelets drop to critically low levels with reported fatality about 5\% [32]. The more severe complication with higher fatality rate reported in the literature is the dengue shock syndrome DSS when increased vascular permeability induced by $\mathrm{T}$ cell released cytokines and plasma leakage cause intravascular depletion with tissue hypo-perfusion and shock [1],[2],[3],[11],[13] with reported fatality up to $44 \%$ [32].

Table of WHO criteria for dengue case definition:

Dengue without warning signs:

-Fever and 2 of the following:

Nausea/vomiting, Rash, Aches and pains, Leukopenia, Positive tourniquet test.

-Dengue (as defined above) with any of the following:

Abdominal pain or tenderness, Persistent vomiting, Clinical fluid accumulation

Dengue with (e.g., ascites, pleural effusion), Mucosal warning signs: bleeding, Lethargy/restlessness, Liver enlargement $>2 \mathrm{~cm}$.

Laboratory: increase in hematocrit concurrent with rapid decrease in platelet count.

-Dengue with at least 1 of the following:

Warning signs require strict observation and medical intervention Severe dengue: Severe plasma leakage leading to shock (dengue shock syndrome) or fluid accumulation with respiratory distress Severe bleeding (as evaluated by a clinician)

Severe organ involvement (i.e., AST or ALT 1000 or greater, impaired consciousness, organ failure).

(CDC page, Dengue case definition. And WHO Dengue case definition.)

\section{Immune Response to Dengue Virus}

After exposure to dengue virus two types of immune response would be initiated: the acute phase called the primary response which is IgM mediated with low titer and secondary response IgG mediated with a higher titer [4].

After infection with one serotype of dengue virus the patient develops immunity to this particular serotype and also cross immunity to other serotypes but this cross immunity is actually short lasting [11]. Unfortunately it was demonstrated that after some time that is not yet well defined if another infection with a different serotype of dengue virus takes place it would induce more severe infection, a phenomenon known as enhancement phenomenon that is proposed to be related to more aggressive $\mathrm{T}$ cell reaction and/or to antibody mediated enhancement through increased virus uptake to the $\mathrm{Fc}$ gamma receptor bearing cells and hence more replication in myeloid cells such as macrophages and monocytes and this 
enhancement phenomenon could be the cause of higher incidence of DSS in patients with secondary dengue infection [3], [4], [5], [6], [11], [14] due to higher number of activated memory $\mathrm{T}$ cells with more release of chemical mediators and hence more aggressive inflammatory response [29].

In a study conducted to study the immune reaction in primary, secondary, tertiary and quaternary dengue infection using ex-vivo model, the secondary infection showed elevated cytokine levels but after tertiary and quaternary challenge there was significant reduction of tumor necrosis factor alpha $\mathrm{TNFa}$, tumor growth factor, Interferon gamma and interleukin 10 [6].

\subsection{Control Measures}

According to the WHO fact sheet updated March 2016 the number of Dengue cases is about 390 million cases worldwide and the number is actually increasing over the last decades [24]. This surge seems to be multifactorial including the unplanned urbanization with dense population settlement in some areas [37], global warming which allows Aedes mosquitoes to survive beyond its usual areas [31], lack of efficient health facilities including sanitary water supply and sewage disposal in addition to deficient control efforts [15], [18] in addition to growing incidence of insecticide resistant mosquitoes [19] which would also limit efficacy of the control efforts as well as international sea and air travel that played an important role in transmission of infection among different nations [17], [21]. It is even expected for dengue infection to reach catastrophic rates within the coming decades due to population growth and climate changes [33]. These facts in addition to the fact that dengue has potential serious complications including mortality of about 22000 annually [31].

All these facts make Dengue a global health burden and challenge to address in particular in absence of a licensed vaccine or specific medical treatment [16].

Dengue control would depend on breaking the loop of events that maintain the Dengue virus and hence spread infection. The loop consists of: the mosquito -bites human to suck blood -lays eggs on stagnant water - eggs hatch giving rise to larvae which grow giving adult mosquitoes. So breaking this circle should limit spread of infection. Most efforts focus on control of vectors due to the fact that wide spread of vectors as well as the four dengue serotypes in same area would result in co-circulation of multiple serotypes which is a warning sign for hyperendemicity as it allows mosquitoes to be carrier for more than one serotype at a time with increased likelihood of severe dengue infections [23], [25].

The insect habitat is mainly in areas deprived from water pipe system as they rely on stagnant water to lay eggs. Example of the habitats such as discarded tires, flower pots, water storage containers also wells, storm drains....etc. [11]. So mosquito control will depend mainly on getting rid of all sources of water stagnation that may serve as habitat for the eggs to be laid and start its life cycle.

\subsection{According to the WHO Statement}

The control will be based on eliminating sources of stagnant water that would complete the loop of the mosquito life cycle through supporting the ova and aquatic phases of the mosquito [36]. Previous experience with programs to control Aedes Aegypti were successful to eliminate the mosquito in the America neotropics but resurgence took place so the WHO is aiming to minimize the load of the vector and the virus as much as possible [36]. So the WHO statement in summary focuses on:

\subsubsection{Environmental Measures}

Infrastructure with adequate water supply and sewage disposal in addition to eliminating sources of stagnant water that would support ova and aquatic phases of vector mosquito, solid waste management and adopting a new construction models that would support the vector elimination efforts such as eliminating roof gutters in new buildings.

\subsubsection{Chemical Measures}

Such as larvicides and insecticides but this measures supposed to be complementary to other more important and safer measures due to potential hazards on humans from these chemicals also growing incidence of insecticide resistance in some areas especially areas where control measures are largely based on insecticides [19], [20].

\section{Conclusion and Recommendations}

Dengue fever affects millions of people annually and it is endemic in tropics and subtropics. Presents with fever, body aches, GI symptoms and headache as the most common manifestations, less commonly skin and chest symptoms.

Mainly affects bone marrow and liver and causes some impressive laboratory changes that could be very helpful for the clinicians to consider the diagnosis including leukopenia and thrombocytopenia and less frequently elevated ALT, but still leukocytosis, normal WBCs count, normal platelets count and normal liver enzymes level are part of the spectrum of dengue fever infection. Therefore, dengue fever is one of the differential in any fever with no clear focus of infection regardless the hematologic picture in particular in endemic areas.

The recovery time for the hematologic and hepatitic changes takes average four days but the hepatitic changes induction and recovery lag behind the hematologic derangement and recovery.

The infection is self-limiting in the majority of cases and the patient would be immune against this particular serotype but still liable for other serotypes infection with even more severe course would be encountered with recurrent infection.

Because of possible co-incidental injurious elements so if elevated liver enzymes persists beyond 9 days with no signs of recovery or markedly elevated enzymes from the start of fever course we recommend to check for other causes such as other viral infections as $\mathrm{HAV}, \mathrm{HBV}, \mathrm{HCV}, \mathrm{CMV}, \mathrm{EBV}$ or other causes of acute hepatitis according to the clinical situation such as drug induced or autoimmune hepatitis.

Due to self-limiting nature of the disease and the benign course in the majority of cases with unavailable anti-viral treatment we recommend conservative treatment as outpatient 
in hemodynamically stable patients that can tolerate oral intake with close monitoring of CBC and ALT every 24-48 hours and stop monitoring once afebrile for at least 24 hours and WBCs and platelets curves started to shift toward recovery.

\section{We Recommend Admission for}

-Hemodynamically unstable patient particularly elderly patients with other co morbidities.

-Severe thrombocytopenia with platelet count below 50,000 due to risk of hemorrhagic complications.

-Progressively rising liver enzymes basically exceeding 10 folds particularly if signs of decompensation such as jaundice or disturbed conscious level.

-In case of fulminant liver failure we recommend transfer to specialized liver center.

At the end we want to stress on the seriousness of the increasing incidence of Dengue infection and this should be addressed at governmental levels with multidisciplinary approach for better outcome.

\section{Limitations of Our Study}

We do not have mortality data because this is retrospective study and files of deceased patients are closed so we cannot detect if the cause of death related to dengue infection or not.

Also number of cases traced for clinical manifestations is less than those traced for laboratory changes because of the nature of this study as retrospective study we found that documentation of clinical presentation and follow up was not appropriate in some cases so we could not include all cases.

\section{References}

[1] www.cdc.gov/dengue/resources/30Jan2012/aegyptifactsheet.pdf

[2] Teoh, E. P., Kukkaro, P., Teo, E. W., Lim, A. P., Tan, T. T., Yip, A., ... MacAry, P. A. (2012). The structural basis for serotype-specific neutralization of dengue virus by a human antibody. Sci Transl Med, 4 (139), 139ra83. http://doi.org/10.1126/scitranslmed.3003888

[3] Sangkawibha, N., Rojanasuphot, S., Ahandrik, S., Viriyapongse, S., Jatanasen, S., Salitul, V., ... Halstead, S. B. (1984). Risk factors in dengue shock syndrome: a prospective epidemiologic study in Rayong, Thailand. I. The 1980 outbreak. American Journal of Epidemiology, 120 (5), 653-669. http://doi.org/10.1093/aje/155.5.478

[4] Midgley, C. M., Bajwa-Joseph, M., Vasanawathana, S., Limpitikul, W., Wills, B., Flanagan, A., ... Screaton, G. R. (2011). An in-depth analysis of original antigenic sin in dengue virus infection. Journal of Virology, 85 (1), 410-421. http://doi.org/10.1128/JVI.01826-10

[5] OhAinle, M., Balmaseda, A., Macalalad, A. R., Tellez, Y., Zody, M. C., Saborio, S., ... Harris, E. (2011). Dynamics of Dengue Disease Severity Determined by the Interplay between Viral Genetics and Serotype-Specific Immunity. Science Translational Medicine, 3 (114), 114ra128-114ra128. http://doi.org/10.1126/scitranslmed.3003084

[6] SB Halstead, Dengue: Overview and History. DENGUE Chapter 1 Imperial College press.

[7] Halstead, S. B. (2007). Dengue. Lancet. http://doi.org/10.1016/S0140-6736(07)61687-0www.cdc.gov/ dengue/resources/30Jan2012/aegyptifactsheet.pdf

[8] Bhatt, S., Gething, P. W., Brady, O. J., Messina, J. P., Farlow, A. W., Moyes, C. L., ... Hay, S. I. (2013). The global distribution and burden of dengue. Nature, 496 (7446), 504-507. http://doi.org/10.1038/nature12060

[9] WHO2016DengueandsevereDengue http://www.who.int/mediacentre/factsheets/fs117/en/

[10] Kularatne Senanayake A M. Dengue fever BMJ 2015; 351: h4661 Doi: http://dx.doi.org/10.1136/bmj.h4661

[11] Webster, D. P., Farrar, J., \& Rowland-Jones, S. (2009). Progress towards a dengue vaccine. The Lancet Infectious Diseases. http://doi.org/10.1016/S1473-3099(09)70254-3

[12] Tom Clarke Dengue virus: Break-bone fever Nature. Doi: 10.1038/news020415-10.

[13] Kurane, I. (2007). Dengue hemorrhagic fever with special emphasis on immunopathogenesis. Comparative Immunology, Microbiology and Infectious Diseases, 30 (5-6), 329-340. http://doi.org/10.1016/j.cimid.2007.05.010

[14] Ana P. Goncalvez, Ronald E. Engle, Marisa St. Claire, Robert H. Purcell, and Ching-Juh Lai monoclonal antibody-mediated enhancement of dengue virus infection in vitro and in vivo and strategies for prevention, PNAS vol. 104 no. 22, Doi: 10.1073/pnas.0703498104.

[15] Hasan, S., Jamdar, S. F., Alalowi, M., \& Al Ageel Al Beaiji, S. M. (2016). Dengue virus: A global human threat: Review of literature. Journal of International Society of Preventive \& Community Dentistry, 6 (1), 1-6. http://doi.org/10.4103/2231-0762.175416

[16] Yacoub, S., Mongkolsapaya, J., \& Screaton, G. (2016). Recent advances in understanding dengue. F1000Research, 5, F1000 Faculty Rev-78. http://doi.org/10.12688/f1000research.6233.1

[17] Annelies Wilder-Smith, M. D., Ph.D., and Eli Schwartz, M. D. Dengue in Travelers. N Engl J Med 2005; 353: 924-932 DOI: 10.1056/NEJMra041927.

[18] Gubler, D. J. (1998). Dengue and Dengue Hemorrhagic Fever. Clinical Microbiology Reviews, 11 (3), 480-496.

[19] Ishak IH, Riveron JM, Ibrahim SS, Stott R, Longbottom J, Irving H, Wondji CS. (2016). The Cytochrome P450 gene CYP6P12 confers pyrethroid resistance in kdr-free Malaysian populations of the dengue vector Aedes albopictus. $2016 \mathrm{Apr}$ 20; 6: 24707. doi: 10.1038/srep24707.

[20] Basile Kamgang, Sébastien Marcombe, Fabrice Chandre, Elysée Nchoutpouen, Philippe Nwane, Josiane Etang, Vincent Corbe and Christophe Paupy. (2011), Insecticide susceptibility of Aedes aegypti and Aedes albopictus in Central Africa. Parasites \& Vectors 4: 79. DOI: 10.1186/1756-3305-4-79.

[21] Gubler, D. J. (2002). Epidemic dengue/dengue hemorrhagic fever as a public health, social and economic problem in the 21st century. Trends in Microbiology. http://doi.org/10.1016/S0966-842X(01)02288-0 
[22] Kow, C. Y., Koon, L. L., \& Yin, P. F. (2001). Detection of Dengue Viruses in Field Caught Male Aedes aegypti and Aedes albopictus (Diptera: Culicidae) in Singapore by Type-Specific PCR. Journal of Medical Entomology, 38 (4), 475-479. http://doi.org/10.1603/0022-2585-38.4.475

[23] M. A. LORON $\sim$ O-PINO, C. B. CROPP, J. A. FARFA' N, A. V. VORNDAM, E. M. RODRI'GUEZ-ANGULO, E. P. ROSADO-PAREDES, L. F. FLORES-FLORES, B. J. BEATY, AND D. J. GUBLER (1999). Common occurrence of concurrent infections by multiple dengue virus serotypes. American Journal of Tropical Medicine and Hygiene, 61 (5), 725-730.

[24] Gubler, D. J., \& Trent, D. W. (1993). Emergence of epidemic dengue/dengue hemorrhagic fever as a public health problem in the Americas. Infect Agents Dis, 2 (6), 383-393. Retrieved from http://www.ncbi.nlm.nih.gov/pubmed/8012739

[25] Burke, D. S., Nisalak, A., Johnson, D. E., \& Scott McN., R. (1988). A prospective study of dengue infections in Bangkok. American Journal of Tropical Medicine and Hygiene, 38 (1), 172-180.

[26] Scott, T. W., Naksathit, A., Day, J. F., Kittayapong, P., \& Edman, J. D. (1997). A fitness advantage for Aedes aegypti and the viruses it transmits when females feed only on human blood. American Journal of Tropical Medicine and Hygiene, 57 (2), 235-239.

[27] Platt, K. B., Linthicum, K. J., Myint, K. S., Innis, B. L., Lerdthusnee, K., \& Vaughn, D. W. (1997). Impact of dengue virus infection on feeding behavior of Aedes aegypti. The American Journal of Tropical Medicine and Hygiene, 57 (2), $119-125$.

[28] Gubler, D. J., Suharyono, W., Tan, R., Abidin, M., \& Sie, A. (1981). Viraemia in patients with naturally acquired dengue infection. Bulletin of the World Health Organization, 59 (4), 623-630.

[29] Vaughn, D. W., Green, S., Kalayanarooj, S., Innis, B. L., Nimmannitya, S., Suntayakorn, S., ... Nisalak, a. (1997).
Dengue in the early febrile phase: viremia and antibody responses. The Journal of Infectious Diseases, 176, 322-330. http://doi.org/10.1086/514048

[30] Vairo F, Mboera LE, De Nardo P, Oriyo NM, Meschi S, Rumisha SF, Colavita F, Mhina A, Carletti F, Mwakapeje E, Capobianchi MR, Castilletti C, Di Caro A, Nicastri E, Malecela MN, Ippolito G. Clinical, Virologic, and Epidemiologic Characteristics of Dengue Outbreak, Dar es Salaam, Tanzania, 2014. Emerg Infect Dis. 2016 May; 22 (5): 895-9. doi: 10.3201/eid2205.151462.

[31] Pang, E. L., \& Loh, H.-S. (2016). Current perspectives on dengue episode in Malaysia. Asian Pacific Journal of Tropical $\begin{array}{llll}\text { Medicine, } & 9 & \text { (4), } & \text { 395-401. }\end{array}$ http://doi.org/10.1016/j.apjtm.2016.03.004

[32] Rigauperez, J., Clark, G., Gubler, D., Reiter, P., Sanders, E., \& Vancevorndam, a. (1998). Dengue and dengue haemorrhagic fever. The Lancet, 352 (9132), 971-977. http://doi.org/10.1016/S0140-6736(97)12483-7

[33] Hales, S., N, de W., Maindonald, J., \& Woodward, A. (2002). Potential effect of population and climate changes on global distribution of dengue fever: an empirical model. The Lancet, 360, 830-834. http://doi.org/10.1016/S0140-6736(02)09964-6

[34] Martina, B. E. E., Koraka, P., \& Osterhaus, A. D. M. E. (2009). Dengue virus pathogenesis: An integrated view. Clinical Microbiology Reviews. http://doi.org/10.1128/CMR.00035-09

[35] Hadinegoro, S. R. S. (2012). The revised WHO dengue case classification: does the system need to be modified? Paediatrics and International Child Health, 32 Suppl 1, 33-8. http://doi.org/10.1179/2046904712Z.00000000052

[36] Dengue - Guidelines for diagnosis, treatment, prevention and control Publication date: 1 October 2009. WHO reference number: WHO/HTM/NTD/DEN/2009.1

[37] Szreter, S. (2004). Industrialization and health. British Medical Bulletin. http://doi.org/10.1093/bmb/ldh005 